\title{
ECG Biometrics using RNN and CNN
}

\section{CURRENT STATUS: POSTED}

Research Square

David Belo

Universidade Nova de Lisboa Faculdade de Ciencias e Tecnologia

dj.belo@fct.unl.ptCorresponding Author

ORCiD: https://orcid.org/0000-0002-5337-0430

Nuno Bento

Universidade Nova de Lisboa Faculdade de Ciencias e Tecnologia

Hugo Silva

Instituto de Telecomunicacoes

Ana Fred

Instituto de Telecomunicacoes

Hugo Gamboa

Universidade Nova de Lisboa Faculdade de Ciencias e Tecnologia

\section{DOI:}

$10.21203 / \mathrm{rs} .2 .22270 / \mathrm{v} 1$

\section{SUBJECT AREAS}

Biomedical Engineering

\section{KEYWORDS}

Pattern Recognition, Deep Neural Networks, Biosignals, Electrocardiogram, Biometry, Identification, Authentication, Machine learning 
Abstract

Background: Biometric Systems (BS) are based on a pattern recognition problem where the individual traits of a person are coded and compared. The Electrocardiogram (ECG) as a biometric emerged, as it fulfills the requirements of a BS.

Methods: Inspired by the high performance shown by Deep Neural Networks(DNN), this work proposes two architectures to improve current results in both identification and authentication: Temporal Convolutional Neural Network (TCNN) and Recurrent Neural Network (RNN). The last two results weresubmitted to a simple classifier, which exploits the error of prediction of theformer and the scores given by the last.

Results: The robustness and applicability of these architectures were tested onFantasia, MIT-BIH and CYBHi databases. The TCNN outperforms the RNNachieving $100 \%, 96 \%$ and $90 \%$ of accuracy, respectively, for identification and $0.0 \%, 0.1 \%$ and $2.2 \%$ equal error rate for authentication.

Conclusions: When comparing to previous work, both architectures reachedresults beyond the stateof-the-art. Even though this experience was a success, the inclusion of these techniques may provide a system that could reduce thevalidation acquisition time.

\section{Full Text}

Due to technical limitations, full-text HTML conversion of this manuscript could not be completed. However, the manuscript can be downloaded and accessed as a PDF.

Figures 


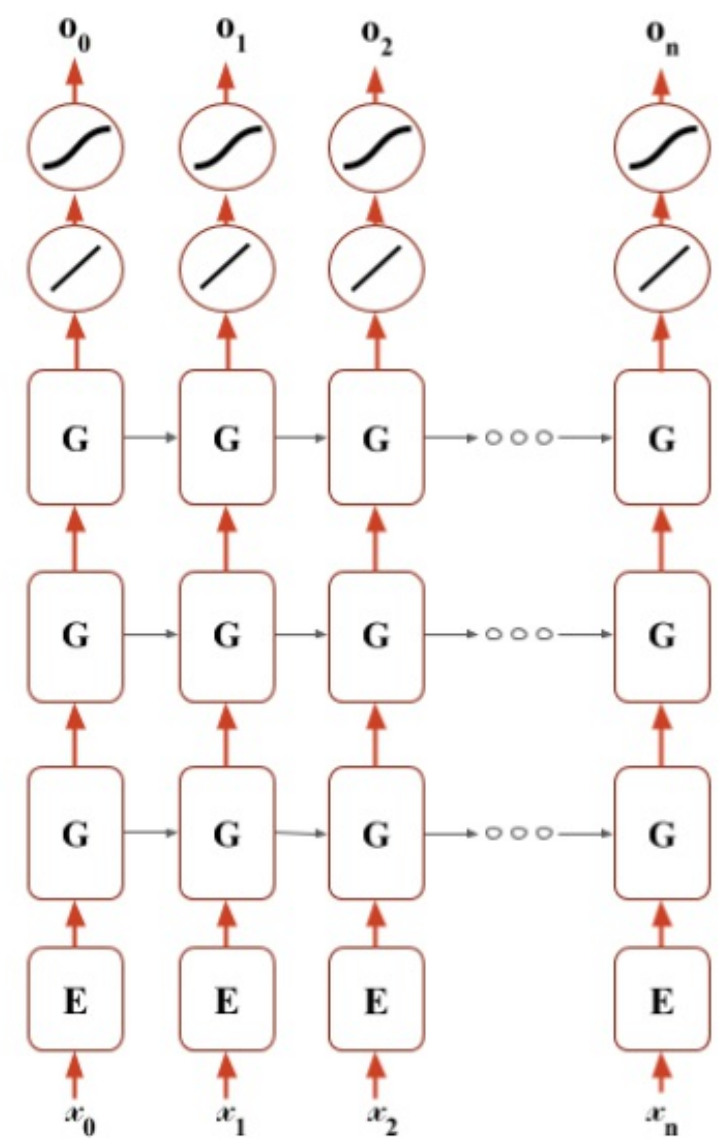

(a) Recurrent Neural Network Approach

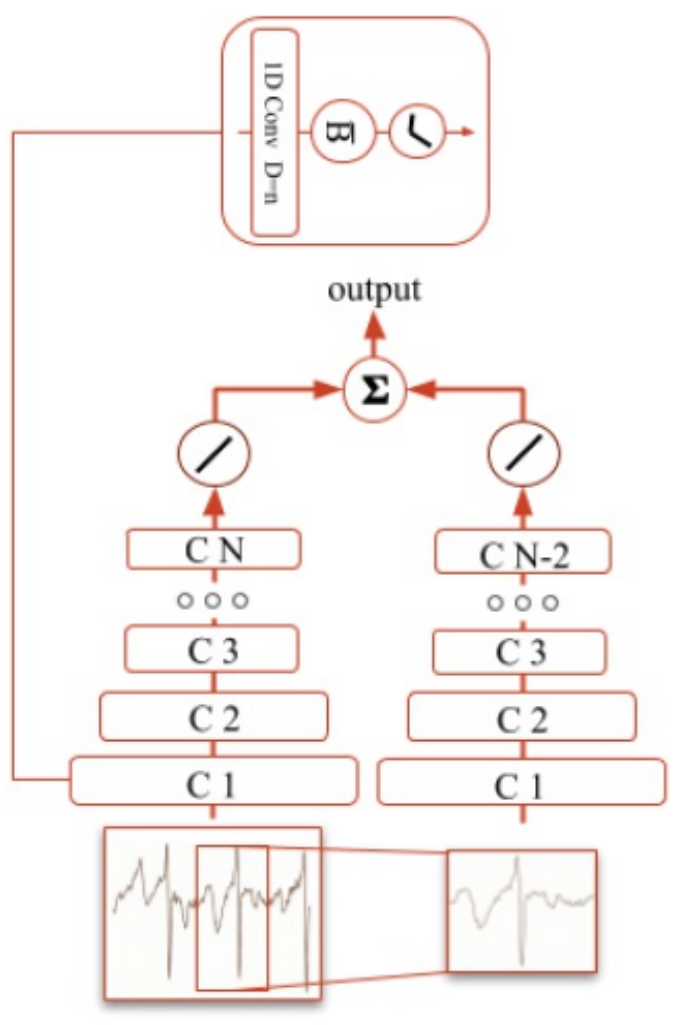

(b) Temporal CNN Approach

Figure 1

Proposed architectures. (1a) depicts the whereRNN approach each input (xn) is fed into to a embedded matrix (E) where the result vector enters into a se- quence of three Gated Recurrent Units (GRU) (G) cells. The output of this sequence is transformed through a fully connected network and a softmax function resulting in on. (1b) depicts the Temporal Convolutional Neural Network (TCNN) approach, where a time window (TW) and the first complete cycle are input to two different sequences of CNN blocks (CN ) each comprised by a one dimensional convolutional layer with $\mathrm{N}$ dilation factor, a batch normalization and a ReLU activation function. One of the networks has $\mathrm{N}$ number of blocks while the other $\mathrm{N}-2$, they both end in a fully connected network and then the last vector is summed giving the output for classification. 


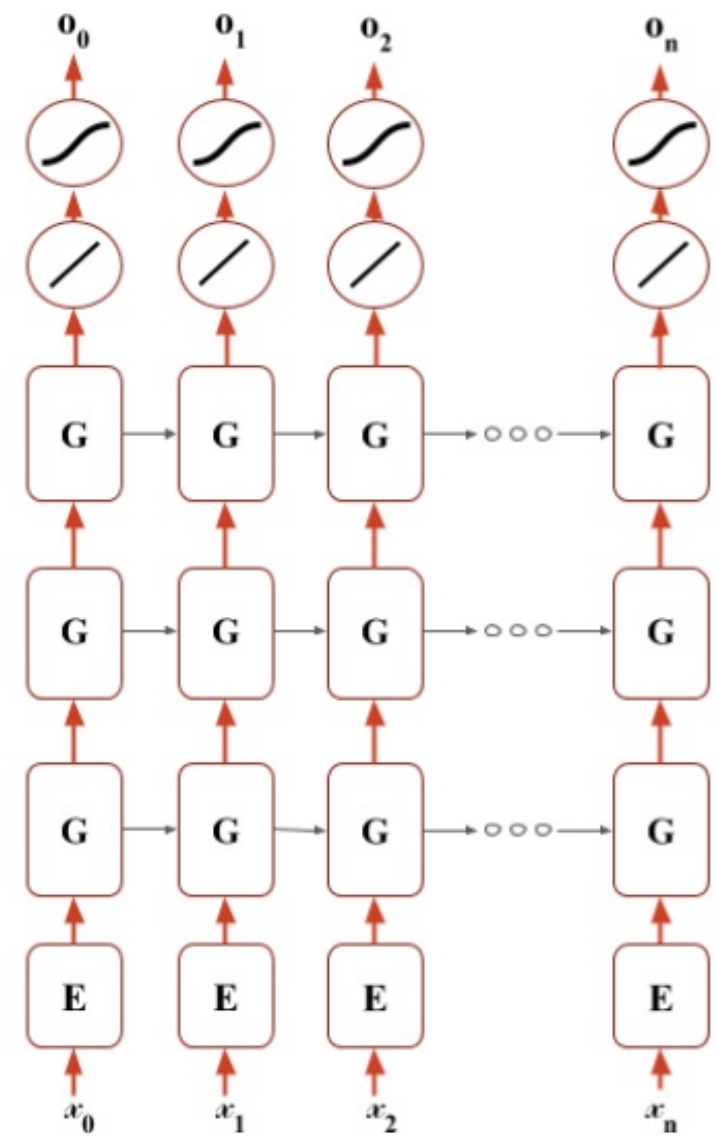

(a) Recurrent Neural Network Approach

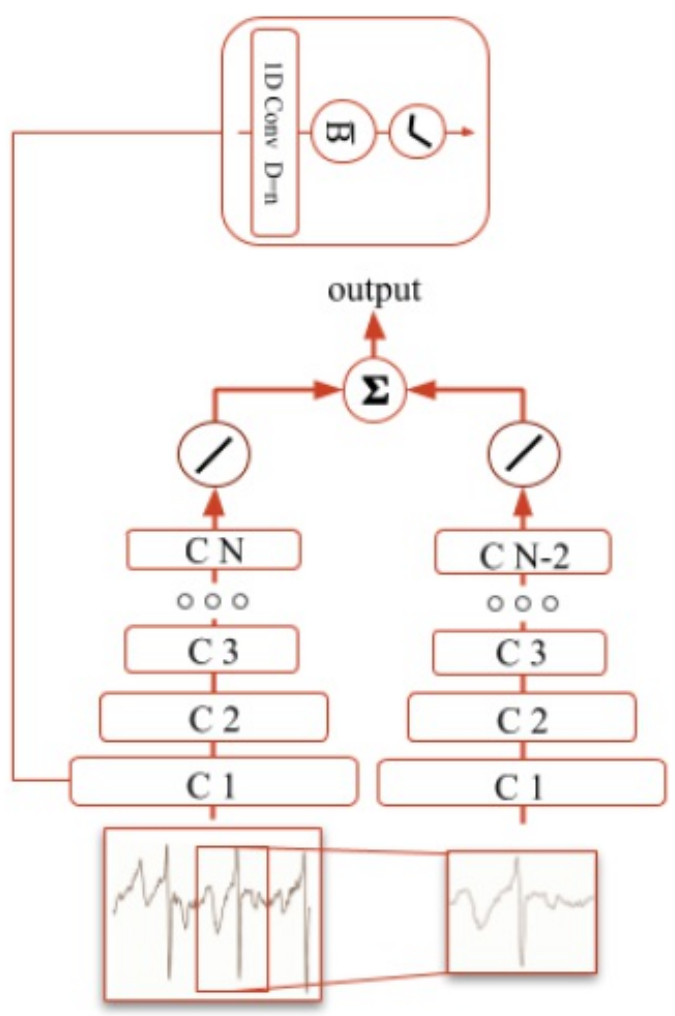

(b) Temporal CNN Approach

Figure 1

Proposed architectures. (1a) depicts the whereRNN approach each input (xn) is fed into to a embedded matrix $(E)$ where the result vector enters into a se- quence of three Gated Recurrent Units (GRU) (G) cells. The output of this sequence is transformed through a fully connected network and a softmax function resulting in on. (1b) depicts the Temporal Convolutional Neural Network (TCNN) approach, where a time window (TW) and the first complete cycle are input to two different sequences of CNN blocks (CN) each comprised by a one dimensional convolutional layer with $\mathrm{N}$ dilation factor, a batch normalization and a ReLU activation function. One of the networks has $\mathrm{N}$ number of blocks while the other $\mathrm{N}-2$, they both end in a fully connected network and then the last vector is summed giving the output for classification. 


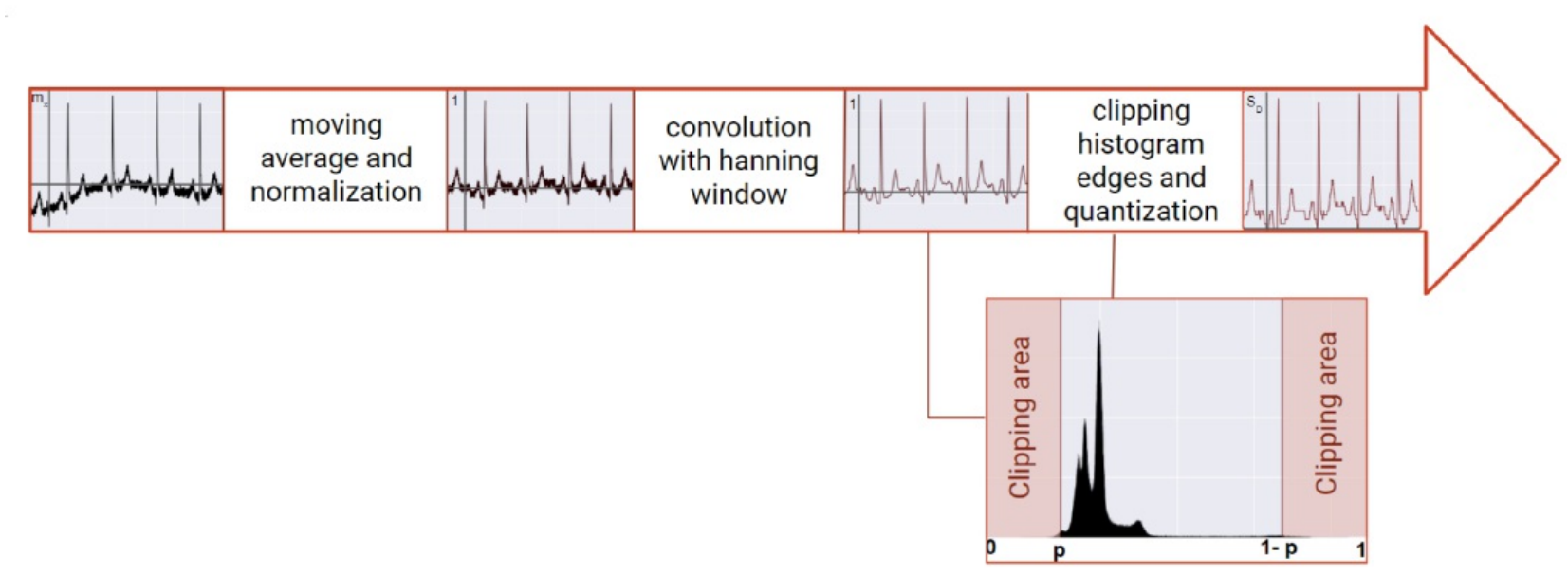

Figure 2

Pre-processing phase for the RNN model.

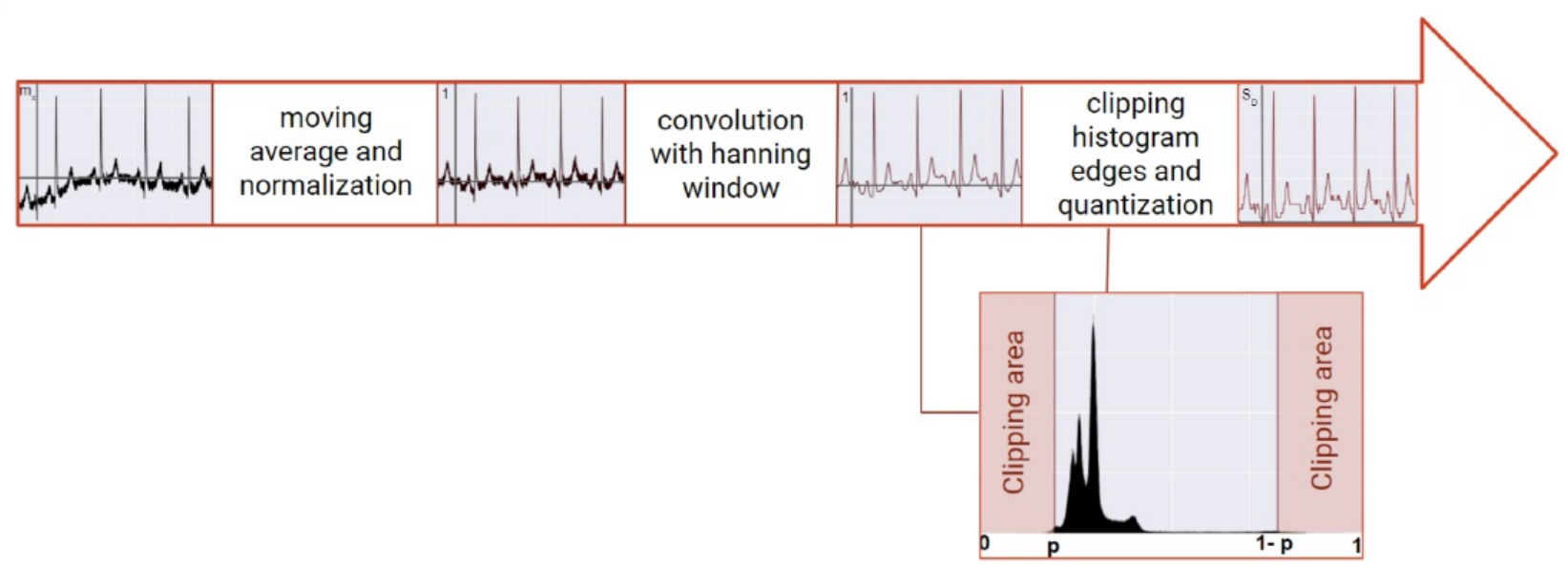

Figure 2

Pre-processing phase for the RNN model. 


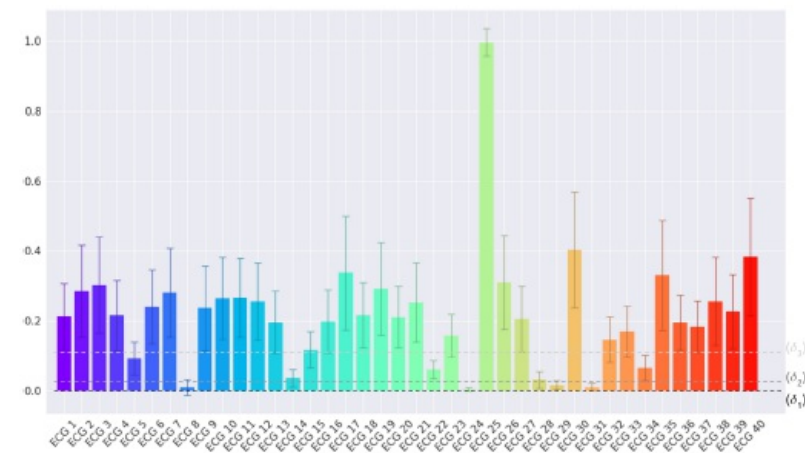

(a) $b=1$

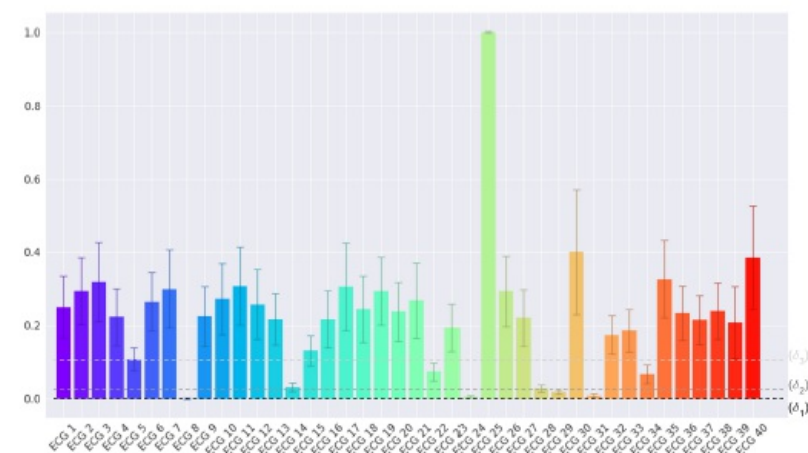

(b) $b=20$

Figure 3

Distribution of the score of the RNN network for the predictor ECG 8 for each of the ECG signals of the Fantasia Dataset. While (3a) refers to a single window of 512 samples, (3b) is using the evaluation for a batch size of 20 windows. $\delta 1, \delta 2$ and $\delta 3$ are three examples of increasing threshold values.

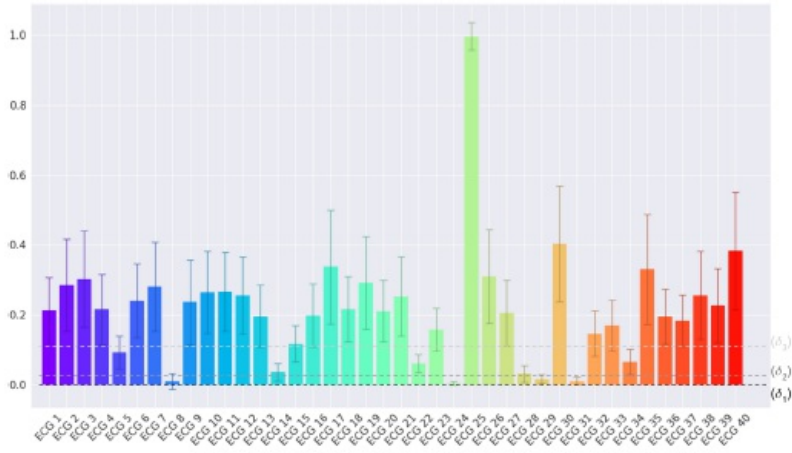

(a) $b=1$

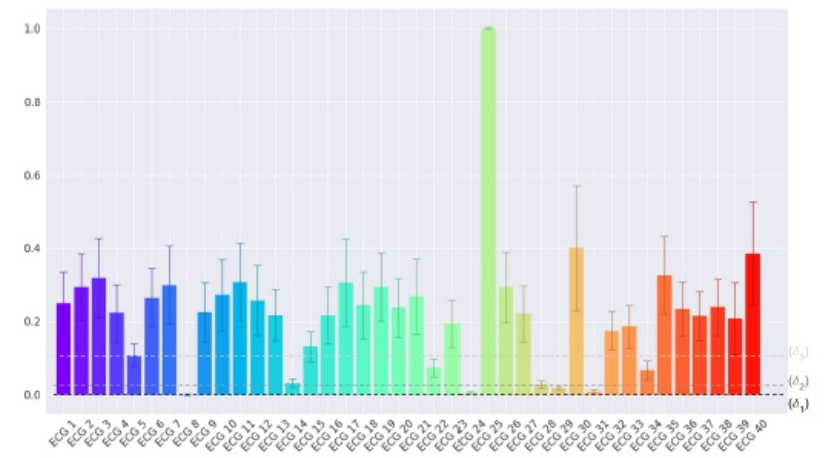

(b) $b=20$

Figure 3

Distribution of the score of the RNN network for the predictor ECG 8 for each of the ECG signals of the Fantasia Dataset. While (3a) refers to a single window of 512 samples, (3b) is using the evaluation for a batch size of 20 windows. $\delta 1, \delta 2$ and $\delta 3$ are three examples of increasing threshold values.

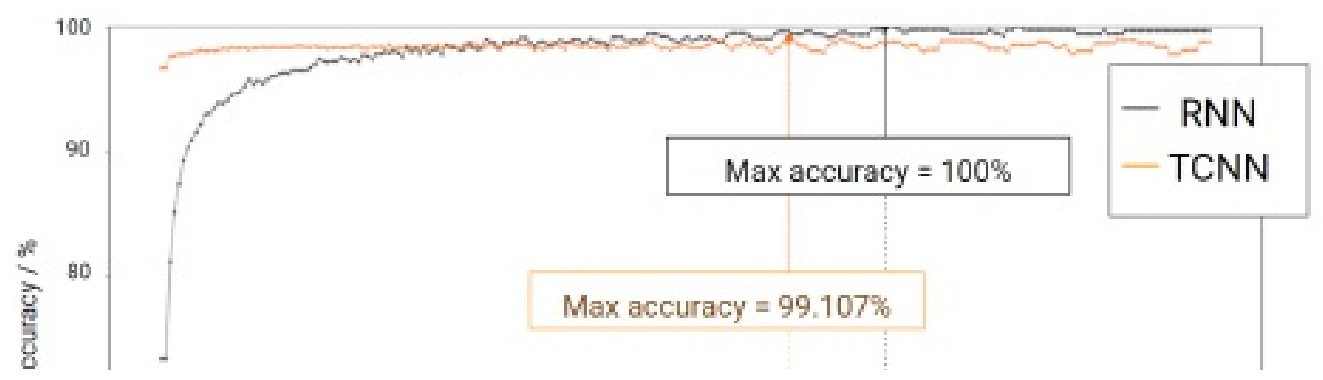




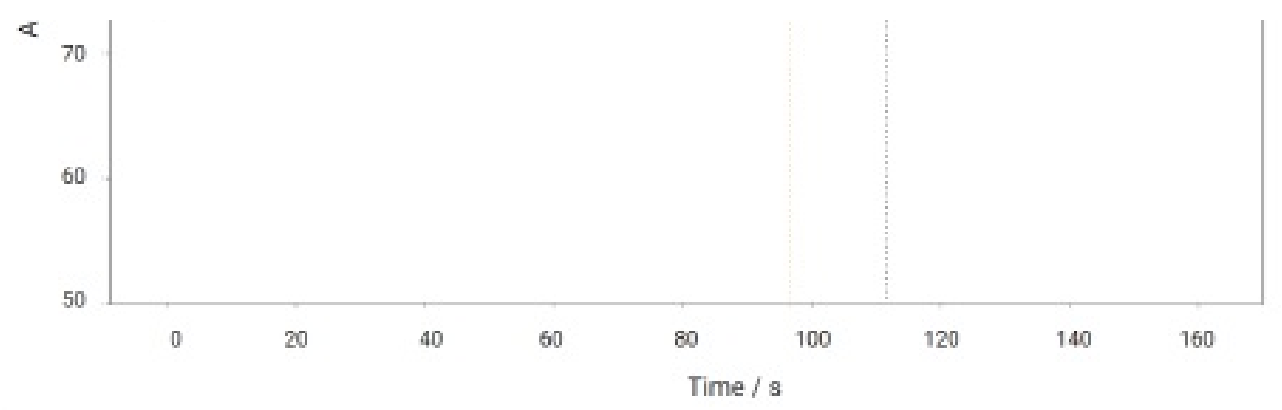

(a)

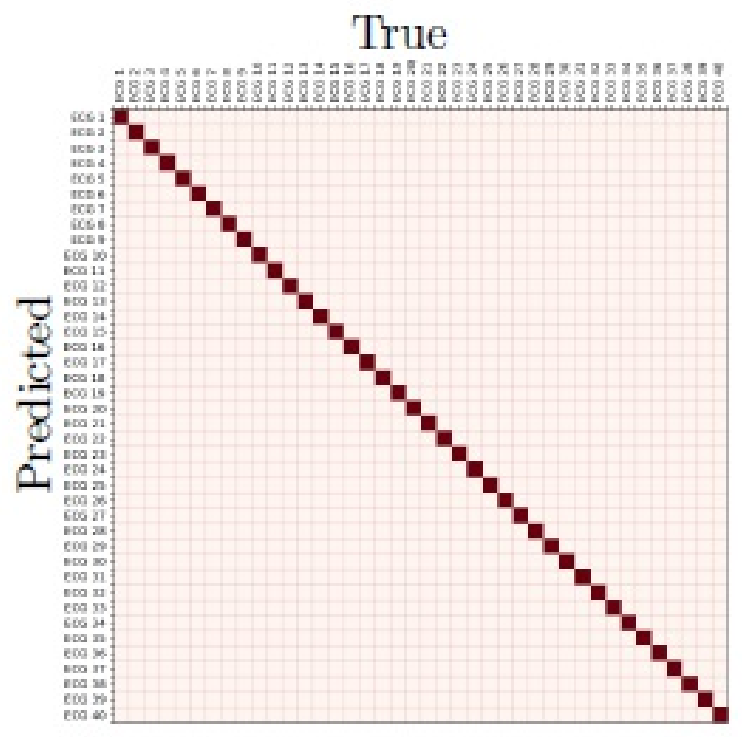

(b) RNN.

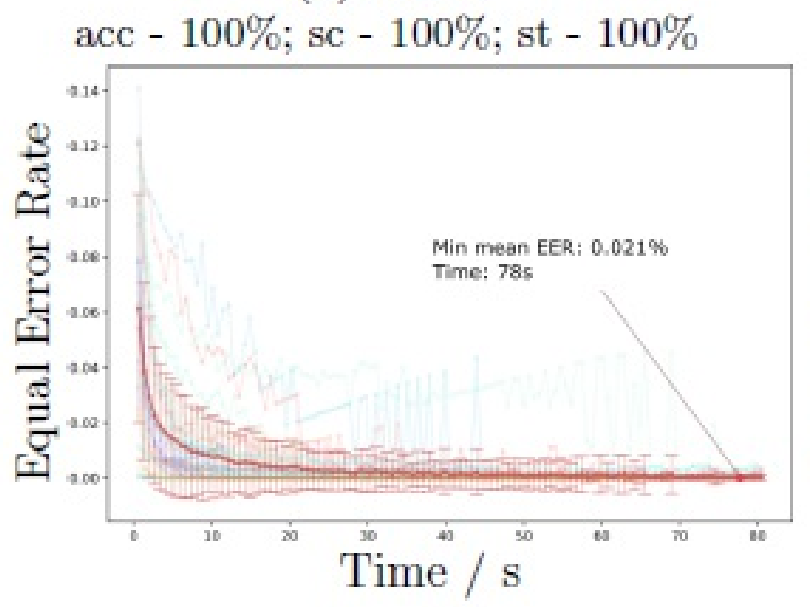

(d) RNN

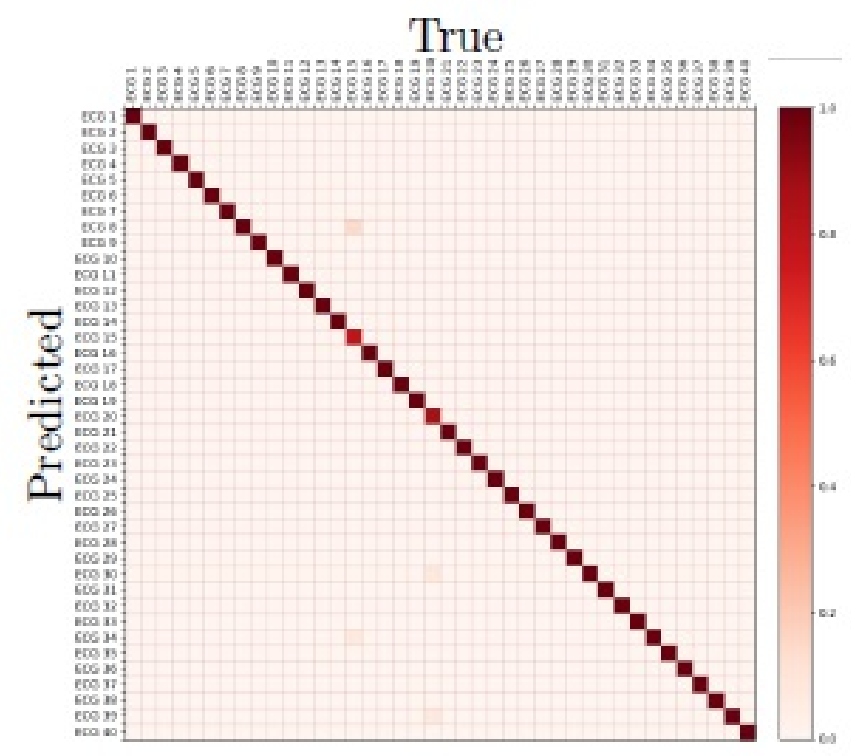

(c) TCNN.

acc $-99.1 \%$; sc $-100 \%$; st $-99.1 \%$

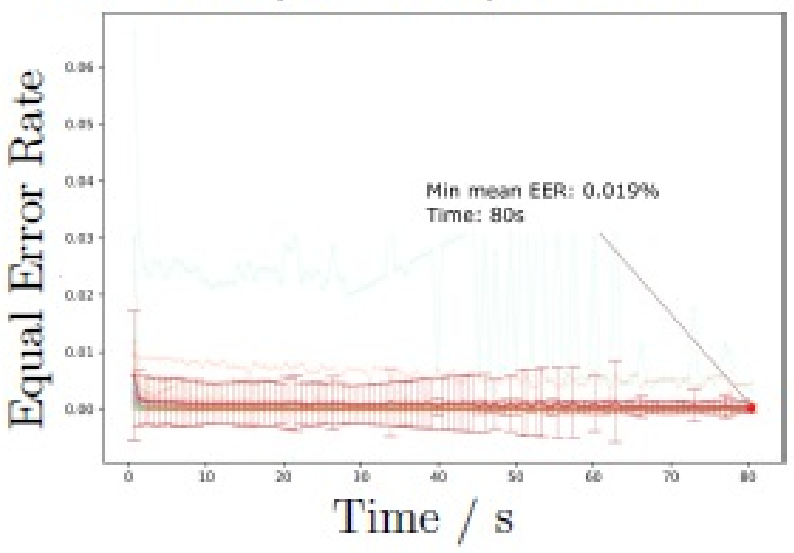

(e) TCNN

Figure 4

Results for the Fantasia dataset. $4 a$ is the identification results with the increase of batch sizes. $4 \mathrm{~b}$ and $4 \mathrm{c}$ are the confusion matrix for the best results for RNN and TCNN approaches, respectively, and respective metrics of accuracy (acc) specificity (sp) and sensitivity (st). $4 d$ 
and $4 \mathrm{e}$ are the evolution of the EER values over time for both approaches. 


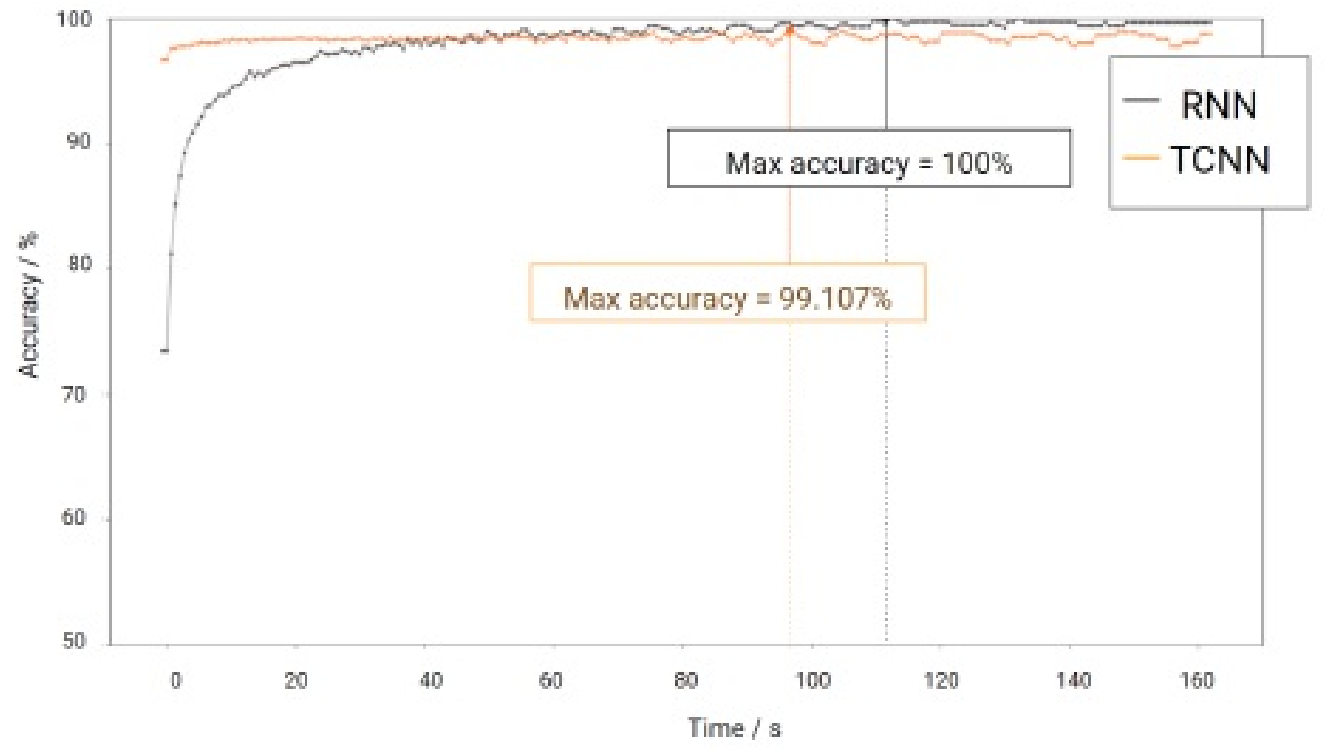

(a)

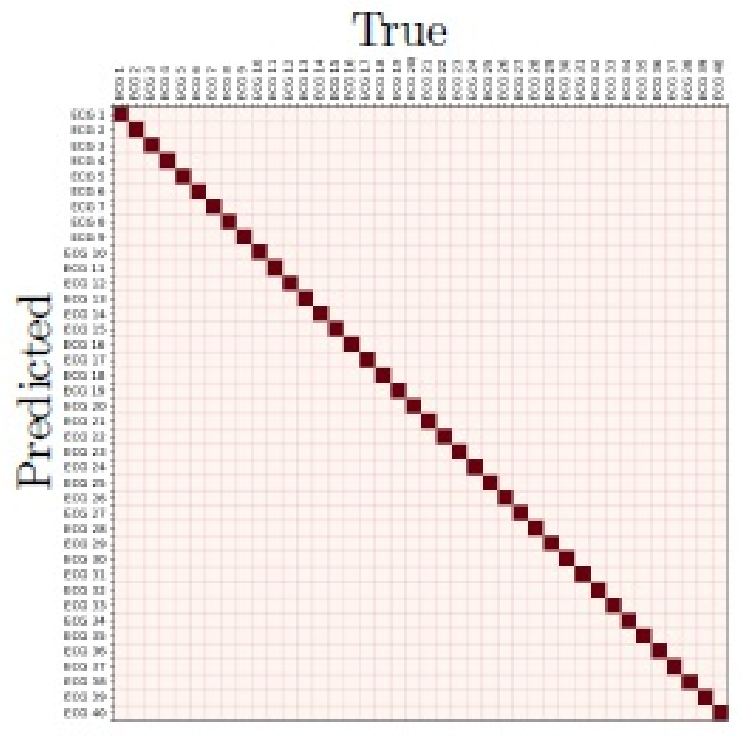

(b) RNN.

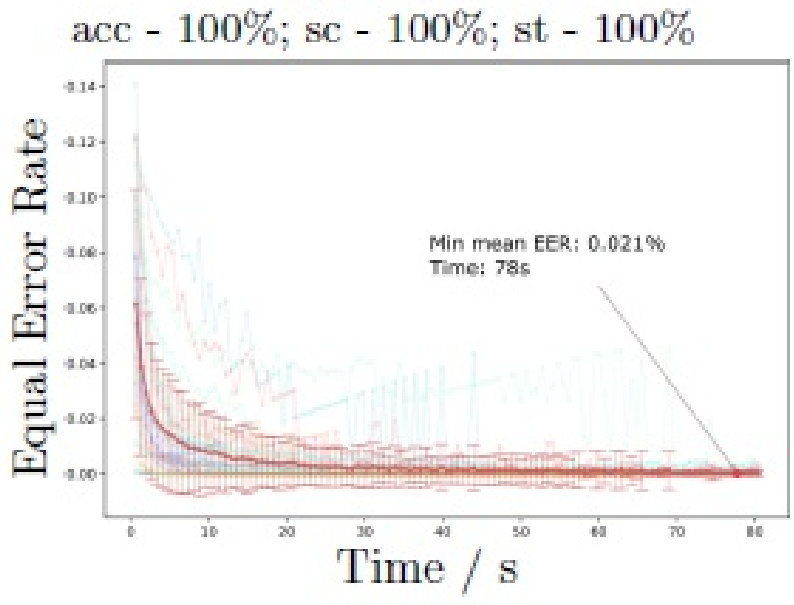

(d) RNN

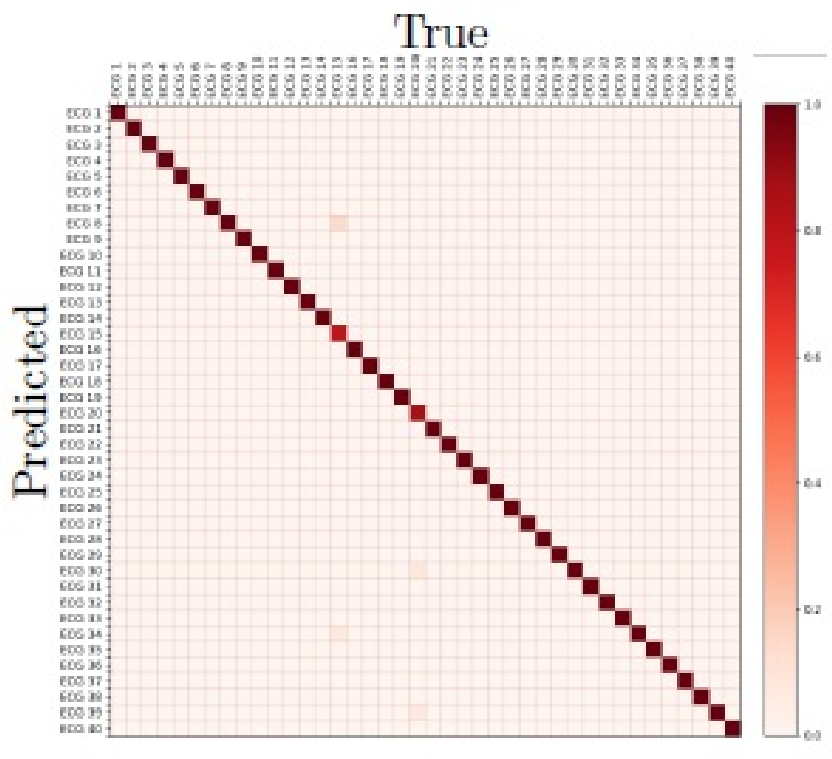

(c) TCNN

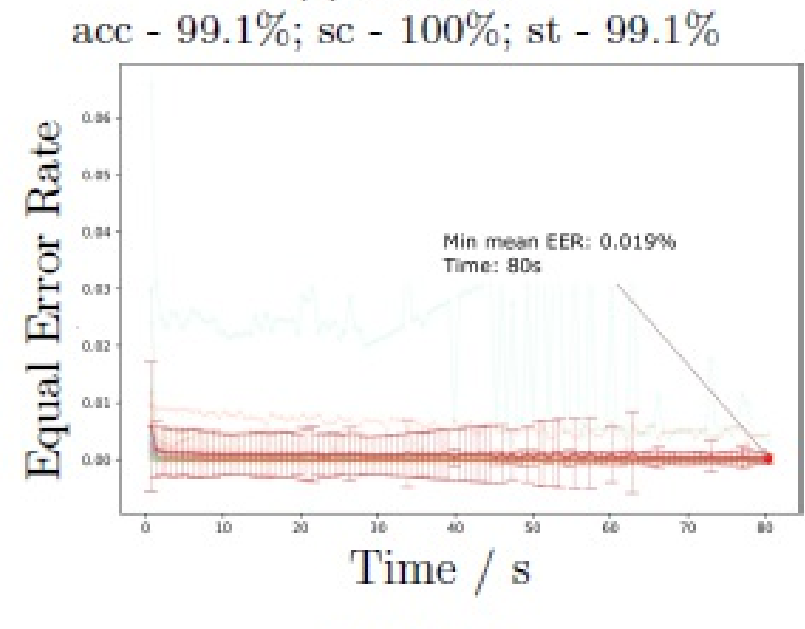

(e) TCNN 
Figure 4

Results for the Fantasia dataset. $4 a$ is the identification results with the increase of batch sizes. $4 \mathrm{~b}$ and $4 \mathrm{c}$ are the confusion matrix for the best results for RNN and TCNN approaches, respectively, and respective metrics of accuracy (acc) specificity (sp) and sensitivity (st). 4d and $4 \mathrm{e}$ are the evolution of the EER values over time for both approaches.

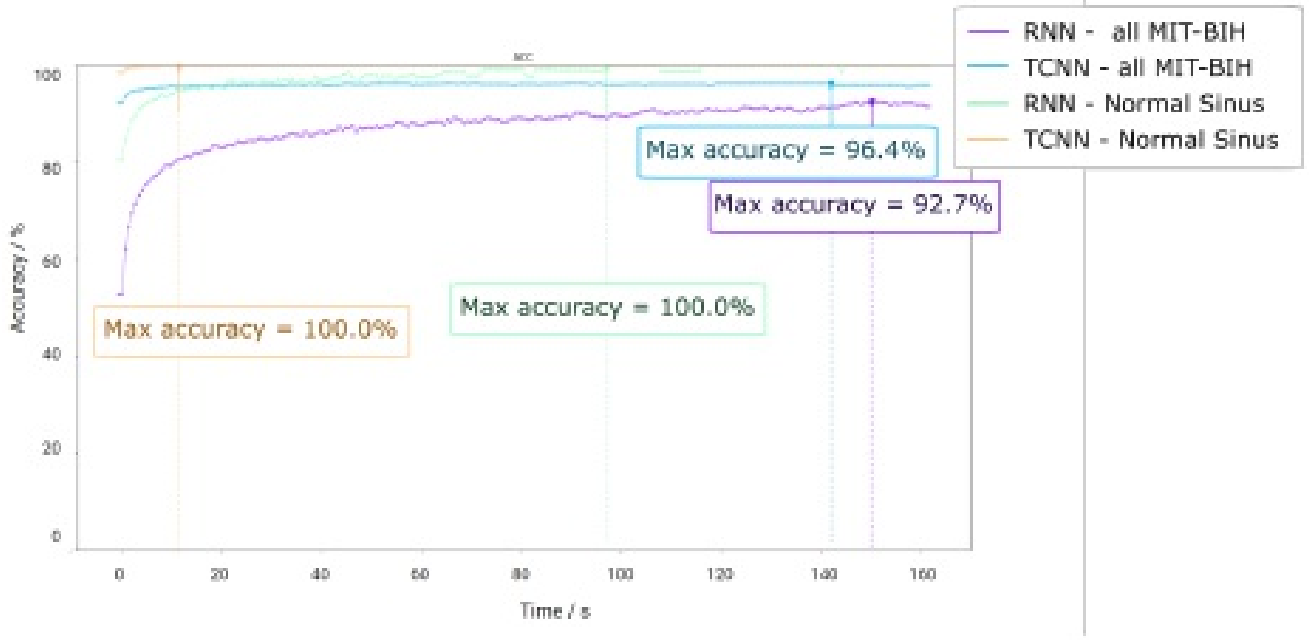

(a)

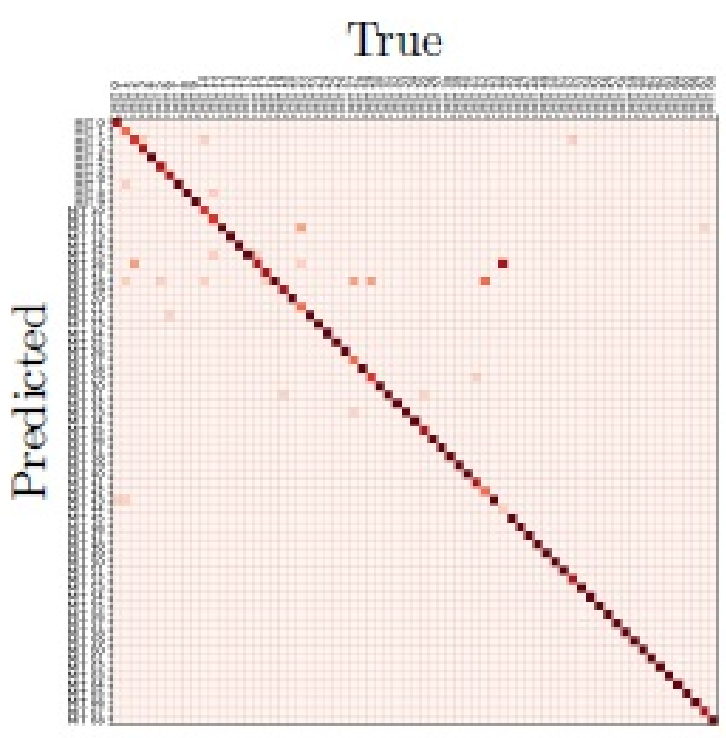

(b) RNN:

acc $-96.4 \% ;$ sp $-99.9 \%$; st $-96.4 \%$

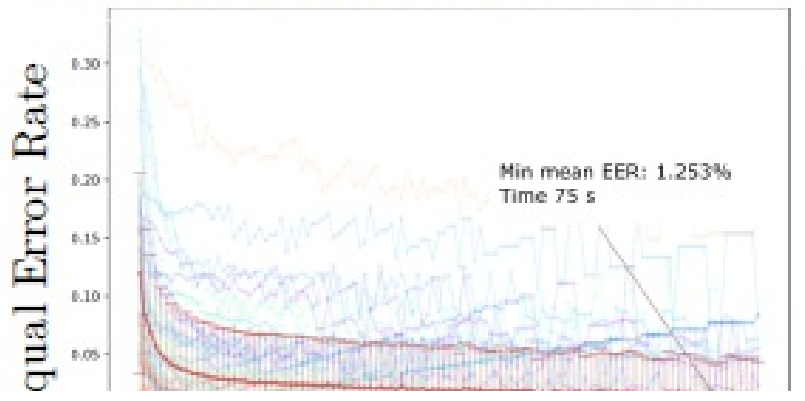

True

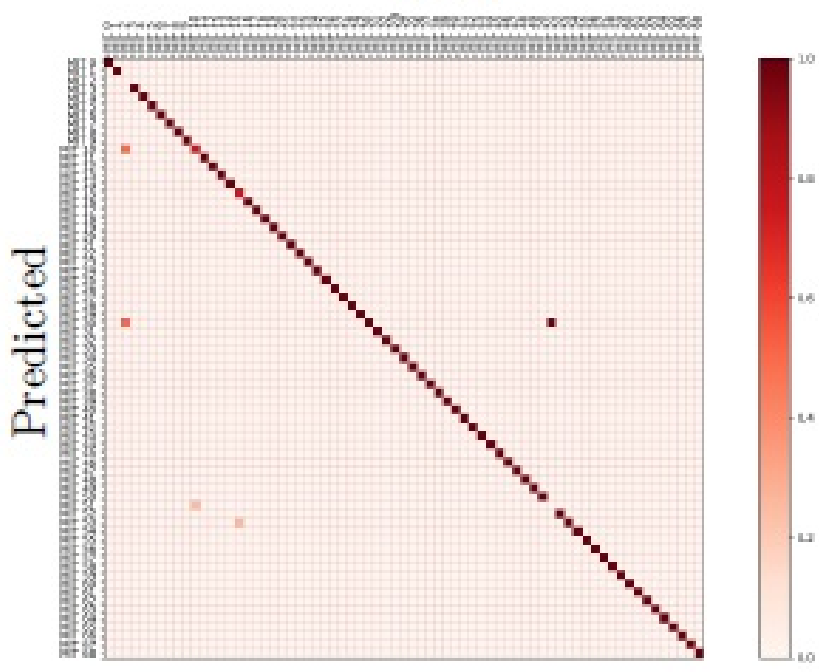

(c) TCNN:

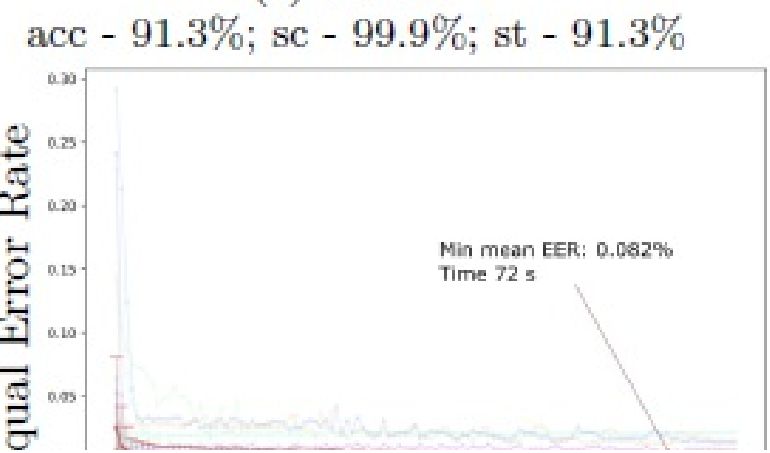




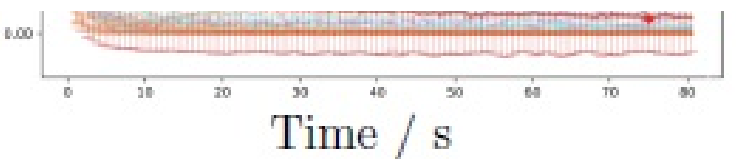

(d) RNN

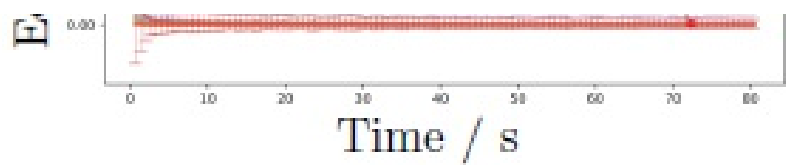

(e) TCNN

Figure 5

Results for the MIT-BIH dataset. $5 \mathrm{a}$ is the identification results with the increase of batch sizes. $5 b$ and $5 c$ are the confusion matrix for the best results for RNN and TCNN approaches, respectively, and respective metrics of accuracy (acc) specificity (sp) and sensitivity (st). $5 d$ and $5 e$ are the evolution of the EER values over time for both approaches.

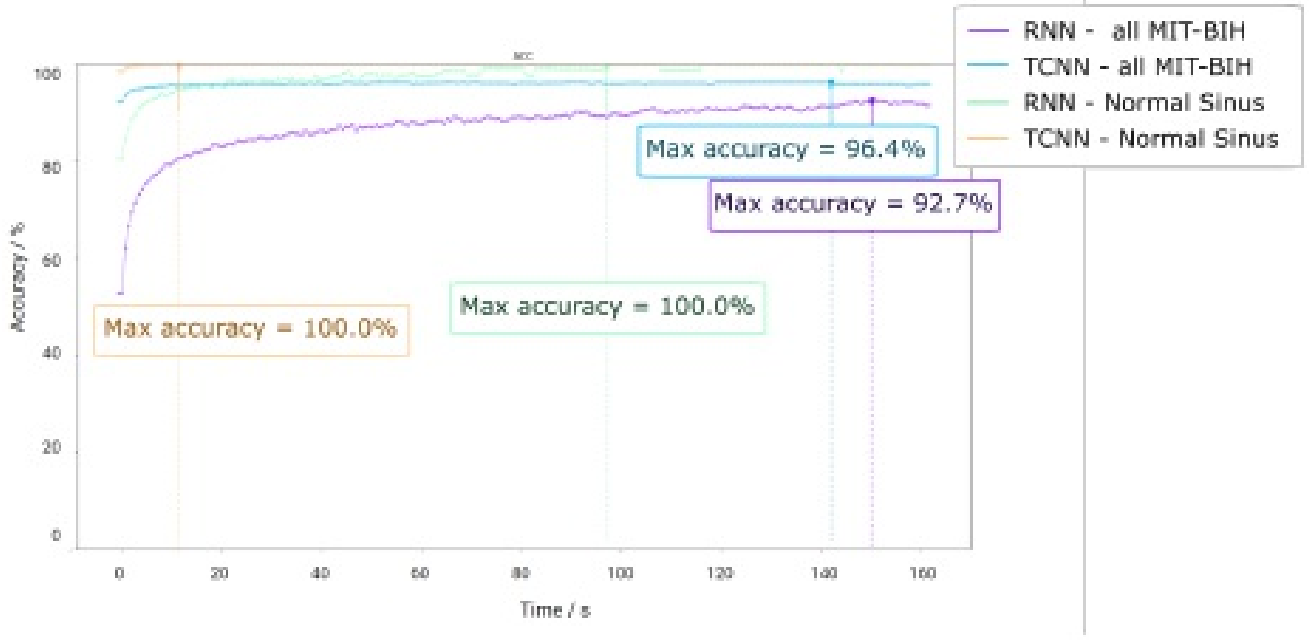

(a)

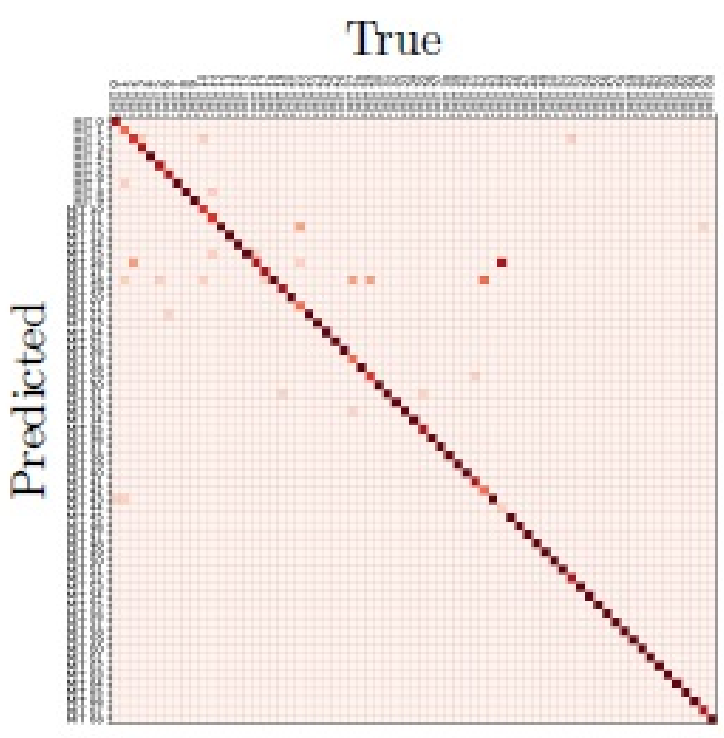

(b) RNN:

acc $-96.4 \%$; sp - $99.9 \%$; st $-96.4 \%$

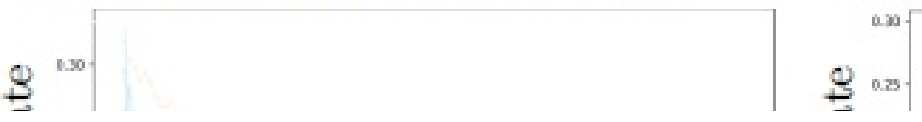

True

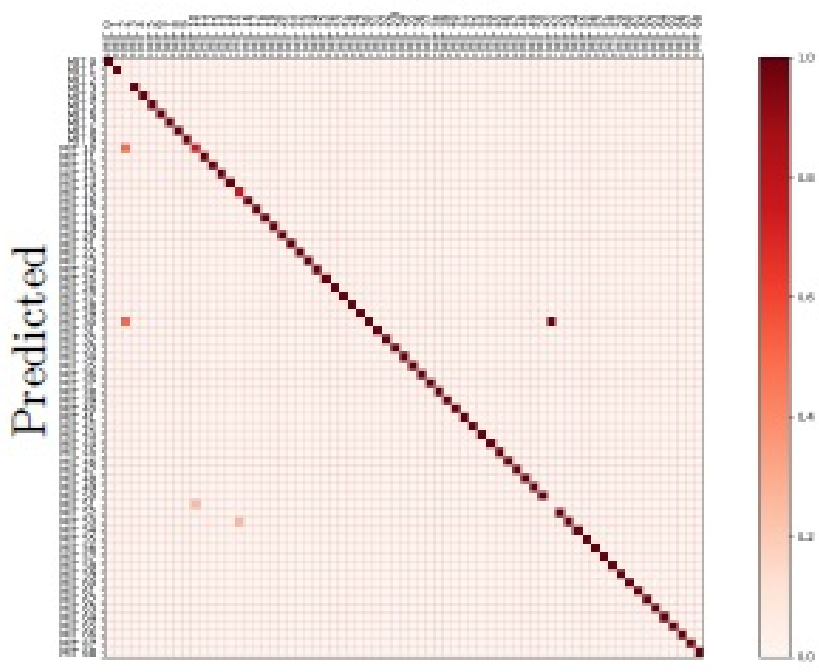

(c) TCNN:

acc $-91.3 \%$; sc $-99.9 \%$; st $-91.3 \%$

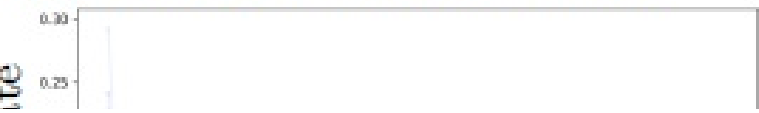




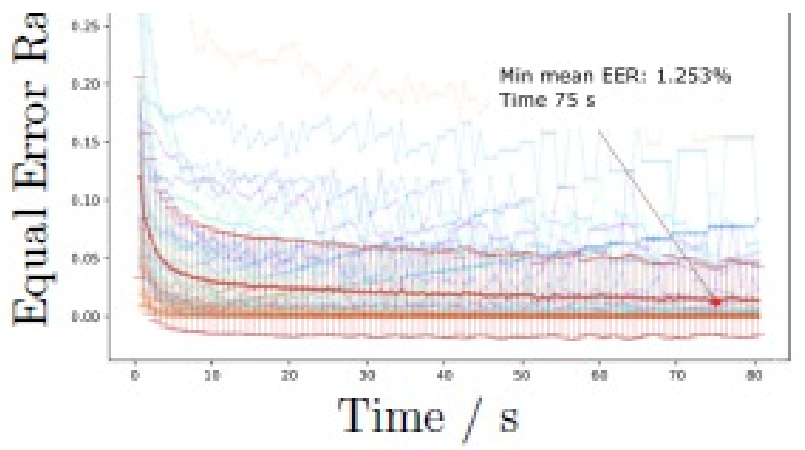

(d) RNN

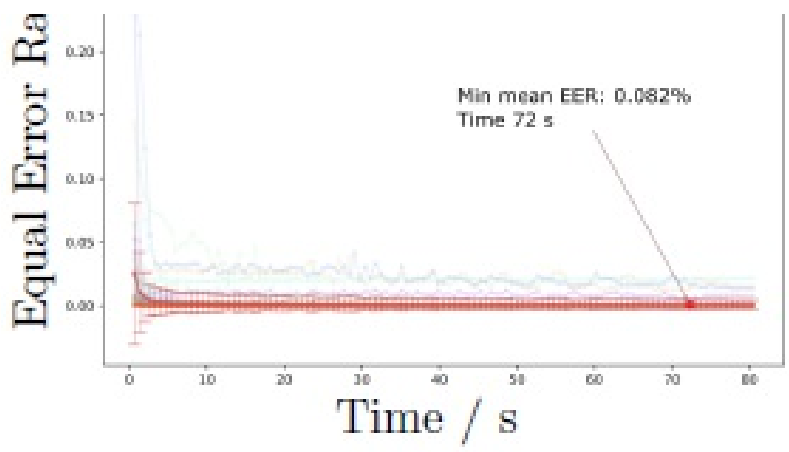

(e) TCNN

Figure 5

Results for the MIT-BIH dataset. $5 \mathrm{a}$ is the identification results with the increase of batch sizes. $5 b$ and $5 c$ are the confusion matrix for the best results for RNN and TCNN approaches, respectively, and respective metrics of accuracy (acc) specificity (sp) and sensitivity (st). $5 d$ and $5 e$ are the evolution of the EER values over time for both approaches. 


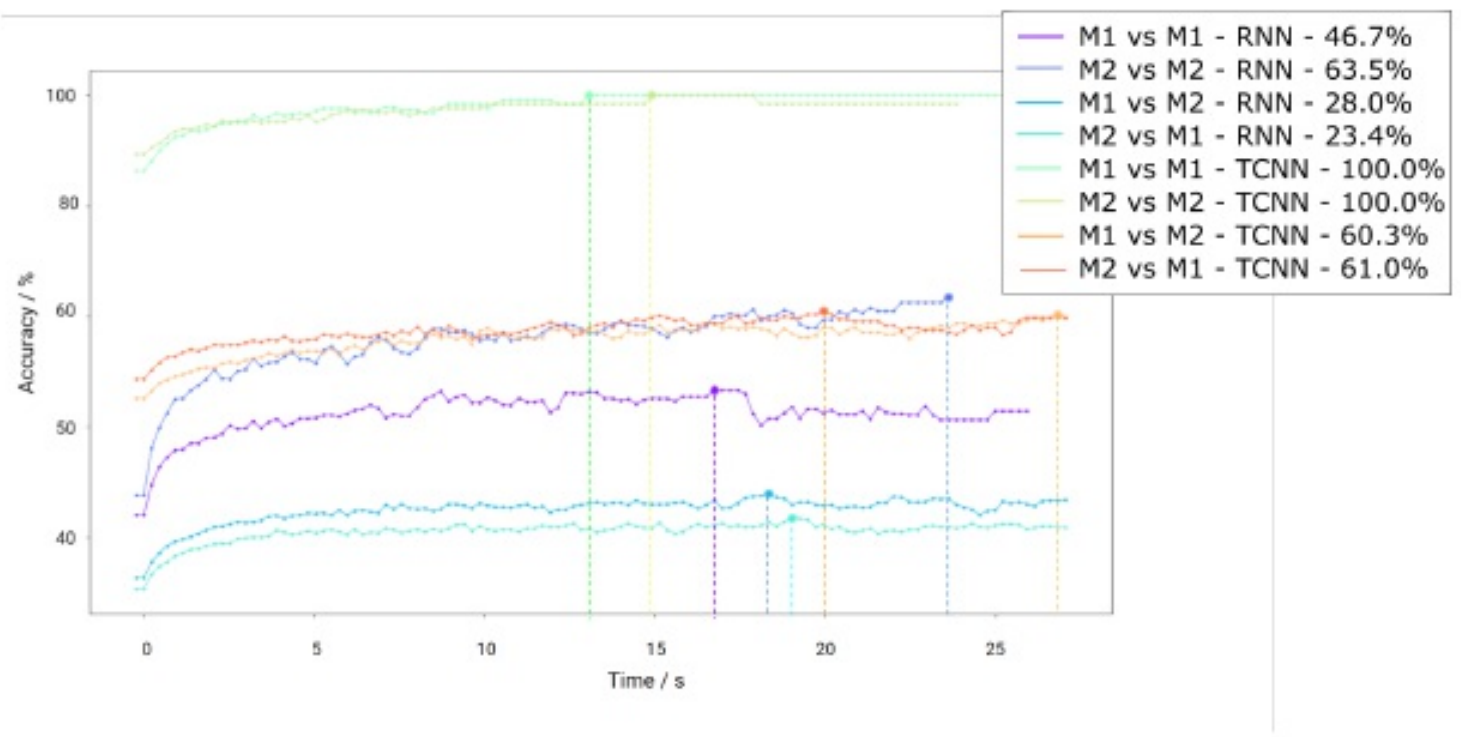

(a)

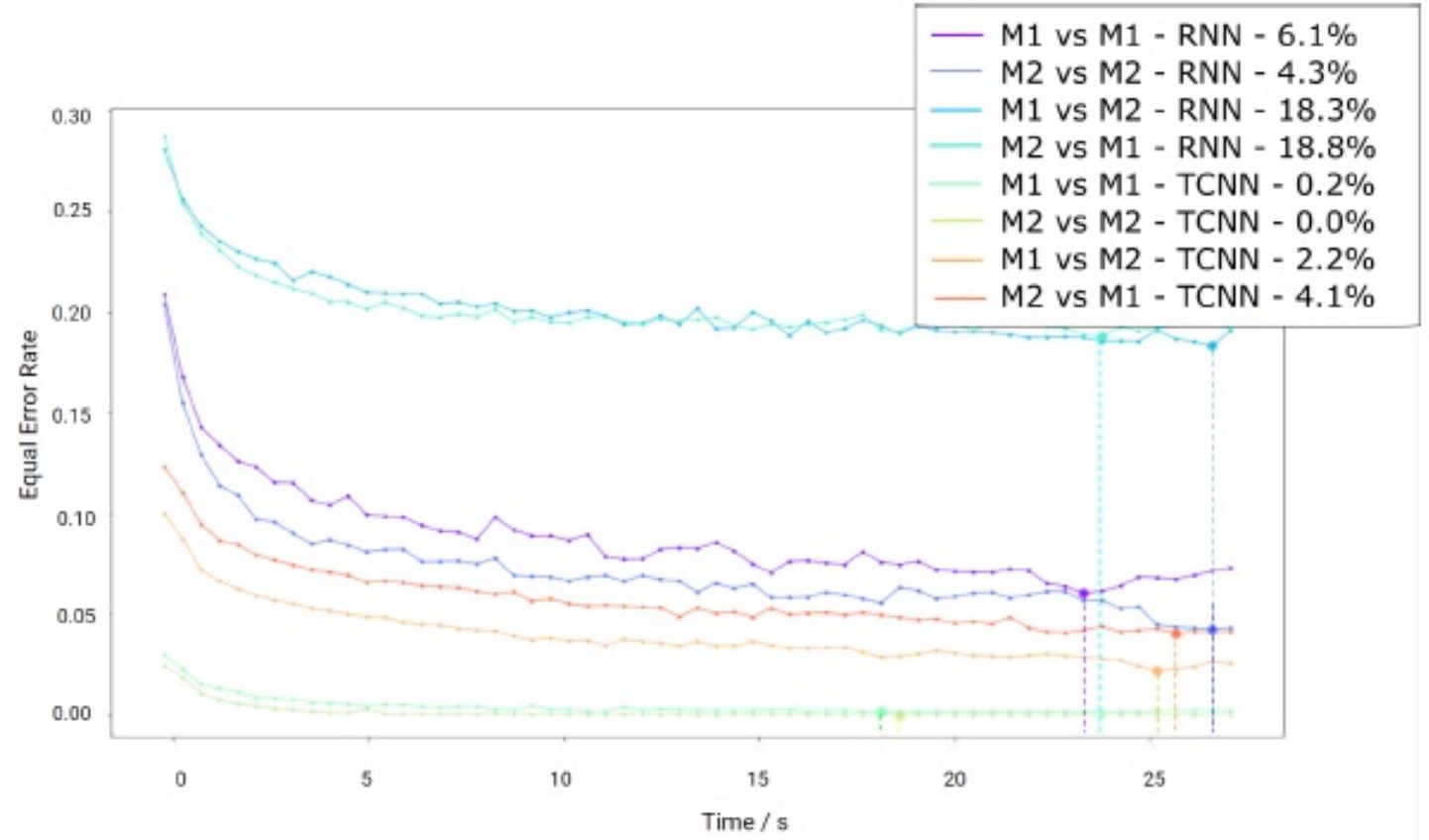

(b) EER values through time.

Figure 6

Results for the CYBHi dataset for each approach. M1 represents the first moment and M2 represents the second moment. 


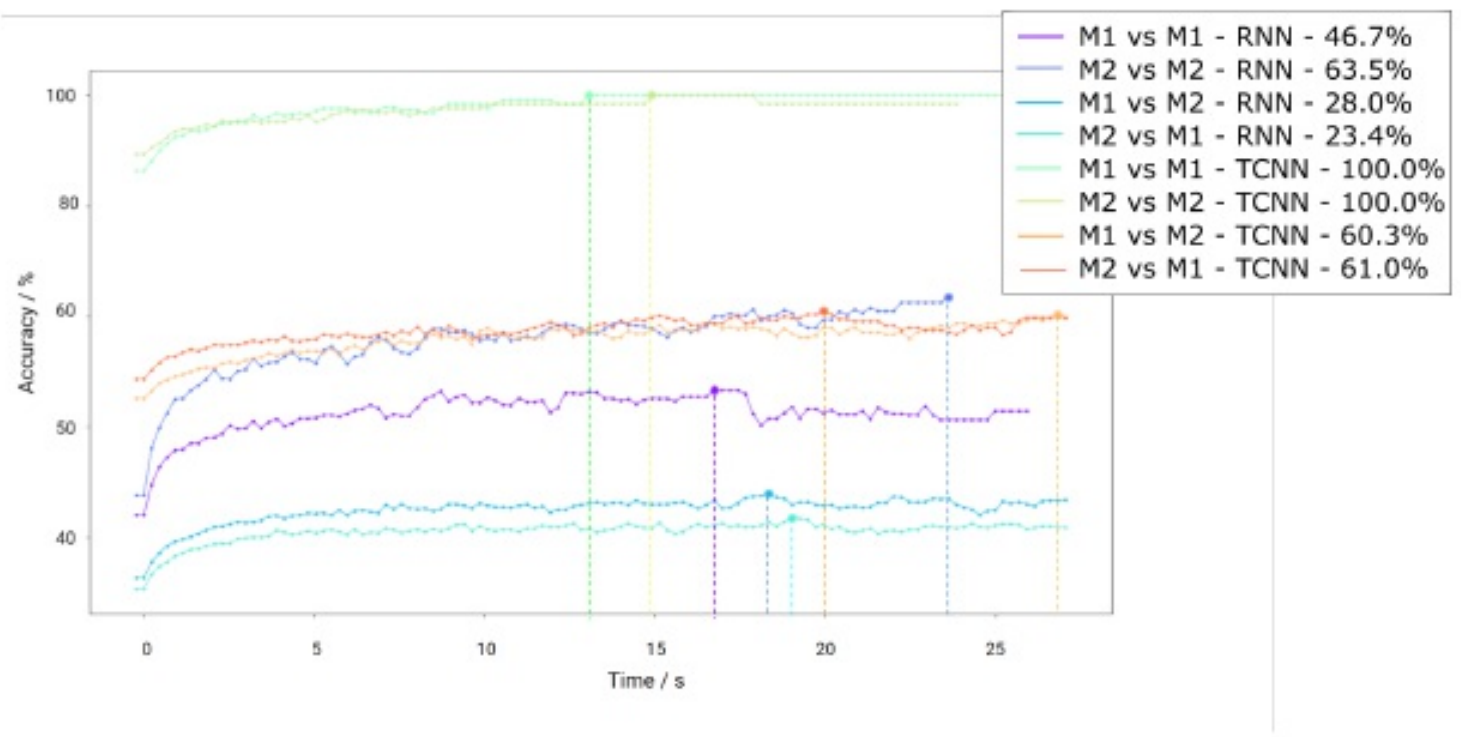

(a)

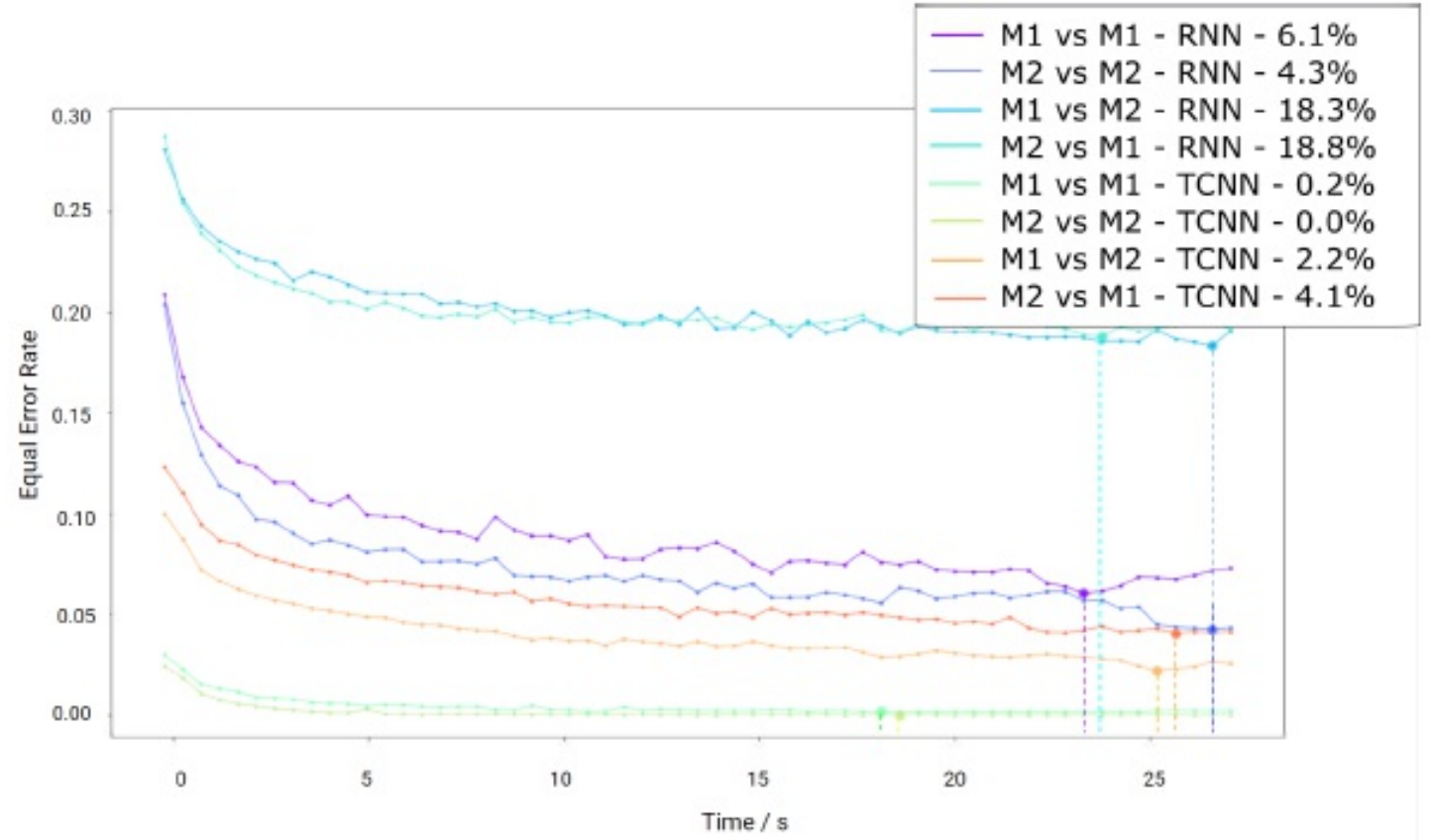

(b) EER values through time.

Figure 6

Results for the CYBHi dataset for each approach. M1 represents the first moment and M2 represents the second moment. 